\title{
Image characteristics of a child with autistic spectrum disorder in representatives of the immediate social surrounding
}

\author{
Anna Azikova ${ }^{1}$, Elena Grinina $^{2 *}$, and Marina Konovalova ${ }^{3}$ \\ 1 Regional rehabilitation center for handicapped children and adolescents, 410022 \\ Sanatorniy Proezd, Saratov, Russian Federation \\ 2 Saratov State University, Department of Rehabilitation Technologies, 410012 \\ Astrakhanskaya st., Saratov, Russian Federation \\ ${ }^{3}$ Saratov State University, Department of Correctional Pedagogy, 410012 Astrakhanskaya \\ st., Saratov, Russian Federation
}

\begin{abstract}
The article demonstrates the insufficiently studied differences in the notions of behavioral manifestations in children suffering from autistic spectrum disorder among representatives of their immediate social surrounding, i.e. parents, psychologists and educators. We used methods of structured observation (D. Stott's observation chart), semi-structured interview (Vineland Adaptive Behavior Scale) and a communicative capabilities diagnostic technique adapted by S.E. Gaidukevich, V. Geisler, F. Gotan). It has been established that parents tend to underestimate the symptom-load of dysfunctional behavior in their children compared to the assessments of these disorders by psychologists and educators. We also established that parents assess the development of most adaptive skills in their children higher than psychologists and educators. The study revealed that there is a low degree of consistency in the assessment of communicative abilities of children with autistic spectrum disorder by parents, educators and psychologists. The obtained data allows us to recommend the introduction of the necessary stage in the organization of psychologist's training for work with a family raising a child with ASD: self-reflection of one's own position in assessing behavioral manifestations, as well as coordination of ideas about symptoms of behavioral disorders in the process of planning and conducting developmental work.
\end{abstract}

\section{Introduction}

Development of a contemporary system of psychologist training in Russia imposes requirements, associated with operation of at least two factors: changing the spectrum of characteristics of professional activity objects, as well as revising the conceptual provisions

\footnotetext{
* Corresponding author: elena-grinina@yandex.ru
} 
of psychological assistance. Additionally, the issues of priority of teaching the universal or specialized methods of psychological diagnosis and correction are still debatable.

Most noticeably, there is a certain delay in the development of practical training of psychologists for professional activity, which can be demonstrated using the example of psychological assistance provision to families raising children with autistic spectrum disorder.

In recent years, there has been an increase in the occurrence of autistic disorders throughout the world and a significant change in the scientific understanding of this defect in biology, medicine, psychology, rehabilitation and pedagogy. Foreign studies indicate heterogeneity of ASD, uncertainty of etiology and pathogenesis, absence of unified approaches to diagnosis, correction and therapy. Thus, some researchers see the key problem of children with autism in impaired ability to empathize with others, which makes it difficult to understand intentions, impulses of others [1], while others believe that the root cause of psychological characteristics in autism are difficulties in coping with changes, lack of ability to plan and solve problems due to changing conditions [2]. Thirdly, people with autism are not capable of identifying the relationship between various elements of reality, they do not see the "whole picture" [3] etc. Eventually, the prevailing position among Russian researchers is that deviant behavior of children with ASD is the result of synergistic interaction of various factors - ontogenetic, psychopathological, psychological, biological and social, which create conditions for social maladaptation [4].

Provision of effective psychological assistance to a child with autistic spectrum disorder is practically impossible without psychological support from parents and creation of a special, "helping" atmosphere in the family. Moreover, a psychologist in this situation is not always an expert, but can only serve as a moderator of individual processes in the complex system of functioning of a family raising a child with ASD. The issues of psychological and pedagogical support of pupils with ASD in the educational system are complicated due to the low awareness of most educators about the possibilities and methods of teaching a child with ASD, extremely pronounced individual differences between such children, lack of specialist tutors, etc.

One of the methods of psychologist capacity building, in our opinion, is the development of reflection of their professional position in relation to complex phenomena of professional activity. In particular, understanding of the nature of differences in the formation of the image of a child with ASD among parents and educators, as well as their own professional position.

\section{Empirical study}

As key structural components in the image of a child with autistic spectrum disorder, we selected perceptions concerning typical symptoms of behavior disorders, forms of adaptive behavior and forms of communication that are characteristic of a child with autistic spectrum disorders.

Purpose of the study: to study the differences in the structure of perceptions concerning characteristic behavioral manifestations in children with ASD among representatives of the immediate social environment.

\section{Goals:}

1. To conduct a comparative analysis of assessments of impaired behavior manifestations in primary school students with autistic spectrum disorder from the point of view of parents, psychologists and educators.

2. To conduct a comparative analysis of assessments of the development of adaptive behavior forms in primary school children with autistic spectrum disorder from the point of view of parents, psychologists and educators. 
3. To conduct a comparative analysis of assessments of communicative skills development in primary school children with autistic spectrum disorders from the point of view of parents, psychologists and educators.

4. To formulate recommendations for taking into account the revealed differences in assessments when training a psychologist-educator for practical work with families raising children with autistic spectrum disorder.

\section{Materials and methods:}

The study involved 12 parents (mothers only) of children with diagnosed ASD ( 8 boys and 4 girls, age: 7 - 9 years old, 5 children were in the first grade, 7 children were in second grade; 10 children were studying within the framework of adopted educational program (AEP) 8.3., 2 children were studying in AEP 8.4.; 2 primary school teachers and a psychologist-educator.

We used the following methods in the study:

1. D. Stott's observation chart for school children. This technique presents a structured observation program for studying behavioral and emotional spheres. It includes 198 fragments grouped into 17 symptom complexes of behavioral disorders. An observer who is well acquainted with behavioral manifestations of a child selects the forms of behavior characteristic of a child from the list. Various manifestations present in the behavior of a child are assessed by 1 or 2 points and are transferred into a standard \% scale for each symptom complex. Values from 0 to $20 \%$ indicate a weak quality manifestation, $20-40 \%$ - a noticeable manifestation, $40-60 \%$ - a strong quality manifestation; $60-80 \%$ - a very strong manifestation. Values in the range of $80-100 \%$ indicate heavy pathological features and the need for additional examination. D. Stott's observation chart is one of the most reliable methods for assessing behavioral disorders in school children [13].

2. Vineland Adaptive Behavior Scale, a semi-structured interview which allows to evaluate characteristics of child's performance in the following areas: communication (receptive, expressive and written speech); everyday life skills (personal, domestic and social); socialization (interpersonal relationships, leisure time and coping skills (coping)); motor skills (gross and fine motor skills).

There is an additional section for assessment of non-adaptive (problematic) behavior. A respondent gives a differentiated assessment of each skill according to the level of use by his child: always - 2 points; sometimes or partially - 1 point; never - 0 points.

The scale is a universally recognized method for assessing adaptation of individuals with normal and abnormal development; it shows highly reliable and valid data [14].

3. Method for diagnosis of communicative abilities, adapted by S.E. Gaidukevich, V. Geisler, F. Gotan for diagnosing communicative abilities of children with severe and (or) multiple disorders of psychophysical development. It is a questionnaire aimed at determining the actual form of communication of children with ASD: characteristics of verbal and non-verbal communication; basic communicative functions; interactive behavior, emotional and psychosocial characteristics. The respondent conducts a differentiated assessment of the child's skills in points: 2 points - the skill is formed, 1 point - the skill is in the formation stage, 0 points - the skill is not formed. Currently, various versions of this method are commonly used when working with children with severe forms of developmental disabilities [15]

\section{Results and discussion}

The data obtained using each of the three methods was compared in order to obtain differences in the assessment of behavioral manifestations in a particular child by a psychologist, a parent, and an educator. We calculated average differences in assessment for each scale of each method in order to identify the differences, then the absolute values 
were converted into relative ones to determine the degree of difference. The differences between the assessments of a psychologist and a parent, a psychologist and an educator, a parent and an educator were also calculated.

\section{Analysis of differences in assessment of dysfunctional behavior symptoms.}

Significant differences in the average assessments of dysfunctional behavior symptoms in children with autism by a psychologist and a parent using Stott's observation chart can be seen through the following indicators: lack of trust to new people (DST): $16.5 \%$, depression (D): $23.2 \%$, hostility in relation to adults (HA): $10.7 \%$, restlessness (R): $17.7 \%$, emotional stress (ES): 15.4\%, neurotic symptoms (NS): 19.9\%, mental retardation (MR): $21.1 \%$. Moreover, according to a psychologist, there is a greater symptoms manifestation in all of these scales, which allows to qualify the behavior of a child as dysfunctional.

Significant differences in average assessments of a psychologist and an educator are observed on the self-care (SC) scale: $13.9 \%$, while an educator qualifies these manifestations in a child as significantly more pronounced than a psychologist). Other than that, assessments of behavioral manifestations in children with autistic spectrum disorders by a psychologist and are educator are consistent, and on average, relative differences do not exceed $5 \%$.

Significant differences in the assessments of behavioral manifestations by educators and parents are observed in such indicators as depression (D): $22.8 \%$, withdrawal (W): $19.0 \%$, restlessness (R): 17.1\%, emotional stress (ES) ): 15.1\%, neurotic symptoms (NS): 19.8\%, mental retardation (MR): $18.2 \%$. From all of the mentioned scales, abnormality in a child's behavior from the point of view of an educator can be defined as more pronounced in comparison with the opinion of parents.

Relative consistency in the assessment by a psychologist, an educator and parents is observed by the following indicators: anxiety in relation to adults (AA), anxiety in relation to children (AC), lack of social normativity (asociality) (A), conflict with children (CC), difficult environment (DE), sexual development (SD), diseases and organic disorders (D), physical defects $(\mathrm{P})$, various symptoms of addictive behavior (AB). It should be also noted that assessments based on the last five indicators for most children were zero, i.e. they were not characteristic of these symptom complexes of behavioral disorders.

Based on the data obtained using D. Stott's observation chart, it can be concluded that on the part of the parents there is a lower assessment of strong negative behavioral manifestations of a child with autistic spectrum disorder than on the part of a psychologist and an educator. Most behavioral disorders based on parental assessments can be classified as mild, while according to educator's and psychologist's opinion they can be qualified as "distinct".

\section{Analysis of differences in adaptive behavior assessments.}

Significant differences in average assessments of adaptive behavior skills of children with autism by a psychologist and a parent using Vineland's scale are observed in the following areas: communication (expressive language: 9.4\%; written language: $8.5 \%$ ); everyday life skills (household skills: 13\%); socialization (interpersonal relationships: $10.1 \%$ ). In all cases, a psychologist assesses these skills in a child as less developed than his parents. Receptive communication skills, personal and social skills of everyday life, play and coping behavior, as well as motor development are assessed by a psychologist and a parent of a child with ASD in a more consistent way. Significant differences on the Vineland's scale are noted in the average indicators received from parents and educators. Parents give significantly higher assessments than educators in terms of receptive communication skills (27.7\%), written communication skills (10.9\%), everyday skills (13.6\%), and coping skills (20.2\%) among their children. Assessments of adaptive behavior of a child with ASD on the Vineland's scale between a psychologist and an educator are consistent in almost all scales. Relative consistency in the assessment of a psychologist, an 
educator and parents is observed on the scales of personal and social skills in everyday life, play behavior, gross and fine motor skills of children with autistic spectrum disorders

Analysis of differences in assessments of communicative abilities of children with autistic spectrum disorders.

There are significant differences in assessments obtained using the method for diagnosing communicative abilities, non-verbal (16.1\%) and verbal $(20.1 \%)$ communication skills; interactive behavior (20.1\%), emotional and psychosocial capabilities (13.6\%) observed between parents and a psychologist. In this case, parents assess the non-verbal communication skills, interactive behavior and psychosocial abilities of their children significantly higher than a psychologist, though a psychologist assesses verbal communication skills highly. When comparing the assessments of a psychologist and an educator there are differences in the assessment of basic communicative functions $(10.0 \%)$ and verbal communication (13.6\%) in children with ASD: a psychologist averagely assesses them higher than an educator. Differences in the average assessments by parents and educators are observed in the assessment of non-verbal communication (14.7\%), basic communicative functions (17.3\%), interactive behavior $(22.7 \%)$ and psychosocial capabilities (13.0\%) of children with ASD. On average parents' assessments are higher than educator's assessments. No consistent assessments by psychologists, educators and parents were obtained in assessing communicative abilities of children with autistic spectrum disorder.

When comparing data obtained using the three techniques, we can state that assessment of behavioral manifestations of children with autistic spectrum disorders on the part of the parents, educators and psychologists has a different degree of consistency. When assessing strongly negative symptoms of dysfunctional behavior, parents tend to underestimate their severity. When evaluating adaptive behavior skills and communication skills, parents are more likely to overestimate them in their children compared with the assessments by educators and psychologists. These data make it possible to emphasize the importance of such stage in training psychologist-educators for work with a family raising a child with autistic spectrum disorder, such as self-reflection, professional position, and coordination of assessments of the child's behavioral manifestations in the process of planning and conducting developmental work.

\section{Conclusions}

1. When assessing behavior of children with autistic spectrum disorder, parents underestimate florid symptoms, such as distrust in people, depression, withdrawal, restlessness, emotional instability, neurotic symptoms, compared to assessments by psychologists and educators. Consistency in the assessments of all participants in the study, i.e. psychologists, educators and parents is observed in terms of indicators: anxiety in relation to adults, anxiety in relation to children and asociality, intensity of which is sufficient, as well as in indicators that are generally not characteristic of primary school children with autistic spectrum disorder (conflict with children, sexual disorders, illnesses and organic disorders, physical defects, various symptoms of addictive behavior).

2. When assessing adaptive behavior skills, there are differences in assessments of expressive and written language, everyday skills, and skills for establishing interpersonal relationships. Parents generally assess these skills as more developed ones in their children compared to educators and psychologists. Relative consistency in the assessment of psychologists, educators and parents is observed in assessing the development of individual skills in everyday life, game behavior, gross and fine motor skills of children with autistic spectrum disorder. 
3. When assessing communicative abilities of children with autistic spectrum disorder, there is a diverse inconsistency in the assessments by parents, educators and psychologists in all measurable indicators: non-verbal and verbal communication, basic communicative functions, interactive behavior and psychosocial abilities.

4. When preparing a psychologist-educator for practical work with families raising children with autistic spectrum disorder, it is necessary to take into account possible inconsistencies in assessing behavioral manifestations in children from the point of view of specialists and parents, as well as to take it into account when planning correctional and developmental work.

\section{References}

1. S. Baron-Cohen, The essential difference: Male and female brains and the truth about autism (Basic Book, New York, 2004)

2. S. Ozonoff, S.J. Roger, \& R.O. Hendern, Autism spectrum disorders: A research review for practioners (American Psychiatric Press, Washington, DC, 2003)

3. F. Happe, J.Briskman, \& U. Firth, Journal of Child Psychology and Psychiatry. 42 (3), 299-307 (2001)

4. N.V. Simashkova, E.V. Makushkin, Autistic spectrum disorders: diagnosis, treatment, observation, clinical practice guideline (treatment guideline) (Moscow, 2015)

5. T.N. Vysotiva, Features of parenting for children with atypical autism (SaintPetersburg, 2013)

6. R.A. Turevskaya, S.S. Senin, Psychological problems of a modern family, collection of writings from VIII International research and practice conference, 587-590 (2018)

7. D.G. Kulina, N.V. Yakovleva, Person in a changing world: health, adaptation, development, 1 (16), 21-47 (2017)

8. S.V. Gulmanova, M.D. Konovalova, Social and cultural integration and special education, 290-297 (2015)

9. E.S. Grinina, A.A. Bessonova, Auditorium. 2 (22), 154-157 (2019)

10. V.V. Dvorianinova, L. N. Kasimova, Medical Almanac, 5(56), 169 - 173 (2018)

11. O.S. Peunkova, A.N. Shalkina, Full-service support of education and professional becoming of handicapped people and people with disabilities, 211-215 (2016)

12. N.A. Platokhina, N.N. Abashina, Research and methodological online magazine Concept, 1, 11-21 (2018)

13. I.V. Dubrovina, Workbook for school psychologic (Prosveshenie, Moscow, 1991)

14. L.R. Saifutdinova, Izvestia of Herzen State Pedagogical University of Russia, 45, 418-423 (2007)

15. S.E. Gaidukevich, Education and upbringing of children in the center for correctional developmental education and rehabilitation (Belarusian State Pedagogical University, Minsk, 2008) 